\title{
Influence de la nature de l'aliment sur l'importance des pertes en particules dans la mesure de la dégradabilité in sacco de l'azote des aliments
}

\author{
B Michalet-Doreau
}

Unité de la Valeur Alimentaire, INRA, Theix, 63122 Ceyrat, France

\begin{abstract}
Summary - In sacco dry matter (DM) and nitrogen $(N)$ disappearances without degradation (ie, lost as particles) were measured in 18 concentrates. Mean losses were $17 \%$ DM and $11 \%$, but were larger with oats (40 and $32 \%$ ). They led to a mean overestimation of $N$ degradability by 3.4 points. Overestimation was higher (up to 8 points) when the losses were high, as for oats, or when degradability was low, as for fish meal.
\end{abstract}

Introduction - Dans la mesure de la dégradabilité in sacco de l'azote des aliments, la fraction qui disparaît du sachet sans être dégradée, c'est-à-dire la fraction "perdue" sous forme de particules, doit tendre vers 0 pour que le classement des aliments ne présente pas de biais. Nous avons donc été amenés à mesurer, sur une vingtaine d'aliments, la fraction de matière sèche et d'azote "perdue" à travers les mailles des sachets, le broyage de l'aliment et la taille des pores des sachets étant par ailleurs fixés.

Matériel et Méthodes - La cinétique de dégradation de l'azote dans le rumen a été mesurée in sacco sur 18 matières premières $(4$ céréales, 5 tourteaux, 3 graines de légumineuses et 6 aliments divers) suivant la méthode décrite par Michalet-Doreau et al (1987). Par ailleurs, pour quantifier la fraction non solubilisée et "perdue" à travers les mailles du sachet, des sachets identiques, contenant ces mêmes aliments, ont été placés dans une solution, pendant $1 \mathrm{~h}$, dans un bain-marie à agitation. La solution a ensuite été filtrée, et les filtres séchés à $80^{\circ} \mathrm{C}$ pendant $48 \mathrm{~h}$, puis pesés. La mesure était répétée 2 fois par aliment.
L'azote des aliments, des résidus de sachets après incubation et des filtres a été déterminé par la méthode Kjeldahl. La teneur en $\mathrm{N}$ soluble ( $\mathrm{N}$ solubilisé dans un tampon; Vérité et Demarquilly, 1978) a aussi été déterminée.

Résultats et Discussion - En moyenne $17 \%$ de la matière sèche est "perdue" et, pour près de la moitié des aliments étudiés, ce pourcentage est inférieur à $10 \%$. Avec la luzerne déshydratée, $9 \%$ de la matière sèche est «perdue", soit une valeur très proche de celles ( 9 à $16 \%$ MS) rapportées par Lindberg et Knutsson (1981) sur des foins étudiés dans des conditions analogues. Mais pour l'avoine et la féverole, cette fraction qui disparaît du sachet sans être dégradée est importante (tableau 1). La fraction qui échappe à la dégradation est relativement plus pauvre en azote que la matière sèche restante, de sorte que le pourcentage d'azote "perdu» n'est que de $11 \%$ en moyenne; certaines valeurs restent cependant élevées (32\% de l'azote de l'avoine) (tableau 1).

La fraction de $\mathrm{N}$ dégradée après $2 \mathrm{~h}$ d'incubation est fortement liée à la teneur 
Tableau I. Importance des pertes en particules dans la méthode in sacco et influence sur l'estimation de la dégradabilité théorique de l'azote.

\begin{tabular}{|c|c|c|c|c|c|c|c|}
\hline & \multicolumn{2}{|c|}{$\begin{array}{c}\text { Disparition } \\
\text { sachets } 2 h(\%)\end{array}$} & \multicolumn{2}{|c|}{$\begin{array}{c}\text { Pertes } \\
\text { particules (\%) }\end{array}$} & \multirow[t]{2}{*}{$\begin{array}{c}\text { N soluble } \\
(\% \text { NT) }\end{array}$} & \multicolumn{2}{|c|}{$D T$} \\
\hline & $M S$ & $N$ & MS & $N$ & & - & «pertes» a \\
\hline $\begin{array}{l}\text { Orge } \\
\text { Blé } \\
\text { Avoine } \\
\text { Maïs } \\
\text { Remoulage } \\
\text { Son fin } \\
\text { Lupin } \\
\text { Féverole } \\
\text { Pois } \\
\text { Txb soja } \\
\text { Tx colza } \\
\text { Tx colza chauffé } \\
\text { Tx arachide } \\
\text { Tx tournesol } \\
\text { Pulpe betterave } \\
\text { Luzerne déshyd } \\
\text { Farine poisson } \\
\text { Farine viande }\end{array}$ & $\begin{array}{l}69,8 \\
67,7 \\
50,2 \\
23,9 \\
59,2 \\
64,1 \\
58,9 \\
74,8 \\
71,1 \\
45,7 \\
52,9 \\
36,2 \\
45,0 \\
48,1 \\
27,0 \\
45,7 \\
42,5 \\
30,9\end{array}$ & $\begin{array}{l}55,5 \\
43,5 \\
74,1 \\
16,4 \\
60,8 \\
64,3 \\
86,4 \\
81,1 \\
78,8 \\
31,8 \\
67,0 \\
30,2 \\
38,3 \\
51,5 \\
23,1 \\
46,8 \\
49,2 \\
36,3\end{array}$ & $\begin{array}{r}9,2 \\
25,9 \\
40,5 \\
10,3 \\
24,4 \\
9,7 \\
14,3 \\
60,7 \\
16,5 \\
5,8 \\
16,3 \\
12,7 \\
19,6 \\
6,3 \\
6,3 \\
8,9 \\
6,3 \\
15,0\end{array}$ & $\begin{array}{r}8,6 \\
11,3 \\
32,1 \\
7,3 \\
4,9 \\
8,5 \\
8,8 \\
8,8 \\
16,9 \\
21,5 \\
4,1 \\
40,0 \\
10,0 \\
14,4 \\
11,3 \\
5,4 \\
4,5 \\
3,5 \\
31,8 \\
11,8 \\
11,1\end{array}$ & $\begin{array}{l}25,6 \\
31,2 \\
31,9 \\
10,0 \\
52,2 \\
56,5 \\
77,3 \\
77,5 \\
80,5 \\
12,0 \\
43,7 \\
11,5 \\
31,2 \\
40,6 \\
13,5 \\
33,0 \\
27,0 \\
18,6\end{array}$ & $\begin{array}{l}79,7 \\
74,2 \\
82,5 \\
34,2 \\
80,9 \\
83,1 \\
93,6 \\
91,0 \\
90,5 \\
66,1 \\
81,6 \\
49,4 \\
72,2 \\
77,7 \\
53,8 \\
67,0 \\
45,8 \\
50,5\end{array}$ & $\begin{array}{l}77,8 \\
70,9 \\
74,3 \\
29,0 \\
80,0 \\
81,5 \\
93,0 \\
89,2 \\
87,9 \\
64,6 \\
79,6 \\
41,0 \\
68,7 \\
76,4 \\
51,7 \\
65,8 \\
38,5 \\
44,3\end{array}$ \\
\hline
\end{tabular}

${ }^{a}$ Corrigée pour tenir compte des pertes en particules. ${ }^{b} \mathrm{Tx}$ : tourteaux.

en $\mathrm{N}$ soluble $(R=0,88)$, mais la précision de la prévision est améliorée quand on prend également en compte la fraction de N qui est "perdue" $(R=0,92)$; cette amélioration concerne essentiellement l'avoine pour laquelle $74 \%$ de $N$ initial a disparu après $2 \mathrm{~h}$ d'incubation alors que seulement $32 \%$ de $\mathrm{N}$ a été solubilisé.

$\mathrm{Si}$ on admet que $\mathbf{N}$ "perdu" se dégrade à la même vitesse que $\mathbf{N}$ alimentaire restant dans le sachet, on peut calculer l'erreur induite par ces pertes en particules sur le calcul de la dégradabilité théorique de l'azote (DT). Ces pertes conduisent à une surestimation de la DT de 3,3 points en moyenne. Cette surestimation est d'autant plus importante que la fraction "perdue" est elle-même importante, soit + 1,9 point pour l'orge (avec $9 \%$ de $\mathrm{N}$ "perdu") contre $+8,2$ points pour l'avoine (avec $32 \%$ de $\mathrm{N}$ "perdu»). Mais pour 2 aliments ayant un pourcentage de pertes identique et égal au pourcentage de pertes moyen, soit $11 \%$, l'erreur est d'autant plus importante que la dégradabilité de l'aliment est faible, soit $+6,2$ points pour la farine de viande contre $+3,5$ points pour le tourteau d'arachide.

Lindberg JE, Knutsson PG (1981) Agric Environ $6,171-182$

Michalet-Doreau $B$, Vérité $R$, Chapoutot $\mathbf{P}$ (1987) Bull Tech CRZV Theix, INRA 69, 5-7

Vérité R, Demarquilly C (1978) In: La Vache Laitière. INRA, 143-157 